\title{
Respiratory physiotherapy in patients with Cystic Fibrosis and upper limb deep vein thrombosis
}

\author{
Tomer Israeli ${ }^{1}$, Iris Eisenstadt ${ }^{1}$, David Shoseyov ${ }^{1}$, Shoshana Armoni ${ }^{1}$, Alex Gileles-Hillel ${ }^{1}$, \\ Galit Cremisi $^{1}$, Malena Cohen-Cymberknoh ${ }^{1}$, and Oded Breuer ${ }^{1}$ \\ ${ }^{1}$ Hadassah Medical Center
}

December 28, 2021

\begin{abstract}
We report physiotherapy management of two patients with severe cystic fibrosis (CF) lung disease and upper limb deep vein thrombosis (DVT). These patients were admitted due to a pulmonary exacerbation. Following peripherally inserted central catheters they were diagnosed with an upper limb DVT. Due to their underlying lung disease, physiotherapy was mandatory for improvement. However, the DVT and anticoagulation treatment raised concerns for pulmonary emboli and hemoptysis. A framework for physiotherapy management in these patients, using a set of precautions and restrictions to maintain airway clearance while minimizing risk for pulmonary emboli and hemoptysis, was established. Using these set of instructions, the patients experienced no major adverse event while maintaining sufficient airway clearance to allow respiratory improvement. These precautions were continued until the upper limb DVTs resolved. To our knowledge there are currently no guidelines nor expert opinions available. Therefore, this framework can help guide physiotherapy management.
\end{abstract}

Respiratory physiotherapy in patients with Cystic Fibrosis and upper limb deep vein thrombosis

Tomer Israeli BPT ${ }^{1,2}$, Iris Eisenstadt $\mathrm{MPH}^{1,2}$, David Shoseyov MD ${ }^{2,3}$, Shoshana Armoni $\mathrm{RN}^{2}$, Alex GilelesHillel $\mathrm{MD}^{2,3}$, Galit Cremisi MSc${ }^{1}$, Malena Cohen-Cymberknoh MD ${ }^{2,3}$, Oded Breuer MD ${ }^{2,3}$

\section{Affiliations}

${ }^{1}$ Department of Physiotherapy and ${ }^{2}$ Pediatric Pulmonology Unit and Cystic Fibrosis Center, Hadassah Hebrew University Medical Center, Jerusalem, Israel.

${ }^{3}$ The Faculty of Medicine, The Hebrew University of Jerusalem, Jerusalem, Israel.

Keywords: Cystic Fibrosis, peripherally inserted central catheters line, deep vein thrombosis, physiotherapy, airway clearance therapy.

\section{Corresponding author:}

Tomer Israeli.

Department of Physiotherapy, Pediatric Pulmonology Unit and Cystic Fibrosis Center, Hadassah-Hebrew University Medical Center, 91120 Jerusalem, Israel, Tel: 972-2-5845026, Fax: 972-2-6435897, e-mail: sidhrta@hadassah.org.il

\section{Funding}

This report did not receive any specific grant from funding agencies in the public, commercial, or not-forprofit sectors. 


\section{Declaration of Interest}

No reported conflicts of interest.

\section{Abbreviated title}

Respiratory physiotherapy in patients with CF and upper limb DVT

\section{Dear Editor,}

Physiotherapy and intravenous antibiotics are the mainstay of treatment of pulmonary exacerbations in patients with cystic fibrosis $(\mathrm{CF}) .{ }^{1}$ Intravenous antibiotics are commonly delivered via peripherally inserted central catheters (PICC lines) ${ }^{1-2}$. A relatively frequent complication of PICC lines are deep vein thrombosis (DVT) with a reported Incidence in CF of $~ 4.5 \%^{2}$, similar to non-CF patients. ${ }^{3}$ DVT may lead to life threatening pulmonary emboli $(\mathrm{PE})$ and therefore prevention, early diagnosis and appropriate management are required. ${ }^{4}$

Guidelines for DVT prevention and management are available and mainly discuss the use of anticoagulation ${ }^{4}$ which in patients with CF may increase the risk for hemoptysis, a relative contraindication for physiotherapy ${ }^{1}$. Clinical practice guidelines for physiotherapy management of patients with upper limb DVT are unavailable. However, guidelines for lower extremity DVT state that due to the risk for developing PE, "mobility is contraindicated until intervention is initiated to reduce the chance of emboli". ${ }^{5}$

Thus, in patients with CF and an upper limb DVT it is prudent to remain alert for hemoptysis and to withhold physiotherapy until the risk for PE is reduced. However, withholding physiotherapy during a pulmonary exacerbation, may carry a risk of further respiratory deterioration.

We herein describe our experience with physiotherapy management of two patients with severe CF lung disease, admitted due to a pulmonary exacerbation, in whom a DVT developed adjacent to their PICC line catheter.

Patient A, a 16-year-old male, with DF508/W1282X CFTR genotype, suffering from a severe pulmonary disease with a baseline forced expiratory volume in 1 second (FEV1) of $32 \%$ predicted and recurrent pulmonary exacerbations for which a PICC line was inserted. On March 2019, he suffered a severe pulmonary exacerbation and was admitted. On day $17^{\text {th }}$ of his admission, a DVT in the left subclavian vein, adjacent to the PICC line, was identified following complaints of acute arm and chest pain.

Patient B, an 11-year-old female, homozygous for the DF508 CFTRmutation, suffering from a severe pulmonary disease, with a baseline FEV1 of $26 \%$ predicted, requiring nocturnal non-invasive ventilation and listed for lung transplantation. On November 2019, she was admitted to the hospital with a life-threatening pulmonary exacerbation for physiotherapy and a prolonged course of intravenous antibiotic treatment via a newly inserted PICC line catheter. During her admission, following complaints of chest pain, she was found to have DVTs in the Rt. Jugular subclavian and brachial axial veins.

For both patients physiotherapy was considered crucial.

Standard physiotherapy care in our hospital for CF patients admitted for pulmonary exacerbations includes: A morning session consisting of exercise combined with airway clearance therapy (ACT), an afternoon exercise session at the gym, and an evening ACT session, with either a physiotherapist or by using a high frequency chest oscillator (vest). ACT, in our center, includes a combination of manual chest physiotherapy, breathing exercises (modified autogenic drainage and modified active cycle breathing techniques) and the use of positive expiratory pressure (PEP) devices.

We conducted a multidisciplinary consultation including a pediatric pulmonologist, a hematologist and senior physiotherapy staff from the $\mathrm{CF}$ center and the intensive care units in our hospital. We formed an approach to physiotherapy treatment which we considered both safe and effective (Table 1). The restriction were continued until DVT resolution. 
Table 1. Framework for physiotherapy management of patients with CF and upper limb DVT receiving anticoagulation treatment.

\begin{tabular}{ll}
\hline Concern & Action \\
\hline Hemoptysis & Continue surveillance of sputum characteristics \\
Pulmonary emboli & Avoid active upper limb movements of the affected \\
& side Withhold treatments with High-Frequency \\
& Chest Wall Oscillation device Withhold manual \\
Further respiratory deterioration & techniques on the affected chest \\
& Use fixation techniques on the unaffected chest Use \\
& positive expiratory pressure devices Continue \\
cardiorespiratory exercises
\end{tabular}

Thus, management included breathing exercises, gentle fixation techniques and the use of oscillatory positive pressure devices (Aerobika, Trudell medical, Pari O-Pep); Also, patient B's non-invasive ventilation device and soap bubble games. In addition, mild lower extremity exercises: treadmill, steps and stationary bicycle.

Patient A, following initial respiratory improvement was discharge and continued outpatient treatment. Outpatient physiotherapists were instructed regarding the required management and a senior physiotherapist from our CF center remained in constant communication.

Patient B, suffered several episodes of mild hemoptysis which resolved spontaneously, without requiring treatment modification.

The treatment framework was followed until the DVTs resolved radiologically. Neither patient suffered from $\mathrm{PE}$, both maintained their respiratory condition.

To conclude, we report our experience regarding physiotherapy management in two patients with CF and severe lung disease who were diagnosed with upper limb DVTs.

To our knowledge, there are no existing guidelines nor expert opinion. This is especially relevant in patients with a more severe disease.

We present a framework established following a multidisciplinary discussion. This framework includes a set of simple instructions easily communicable. Adhering to these instructions enabled us to maintain our patients' respiratory status without any major adverse events.

Since physiotherapy is standard treatment in all patients with CF, and especially during pulmonary exacerbation, and since DVT is not an uncommon complication and poses an increased risk for both pulmonary emboli and hemoptysis: specific guidelines are required for management of patients with CF suffering from upper and lower limb DVTs.

\section{References:}

1. Smyth AR, Bell SC, Bojcin S, Bryon M, Duff A, Flume P, Kashirskaya N, Munck A, Ratjen F, Schwarzenberg SJ, Sermet-Gaudelus I, Southern KW, Taccetti G, Ullrich G, Wolfe S; European Cystic Fibrosis Society. European Cystic Fibrosis Society Standards of Care: Best Practice guidelines. J Cyst Fibros. 2014 May;13 Suppl 1:S23-42. doi: 10.1016/j.jcf.2014.03.010. PMID: 24856775.

2. Nash EF, Helm EJ, Stephenson A, Tullis E. Incidence of deep vein thrombosis associated with peripherally inserted central catheters in adults with cystic fibrosis. J Vasc Interv Radiol. 2009 Mar;20(3):34751. doi: 10.1016/j.jvir.2008.11.018. Epub 2009 Jan 20. PMID: 19157904.

3. Nabil Fallouh, Helen M. McGuirk, Scott A. Flanders, Vineet Chopra, Peripherally Inserted Central Catheter-associated Deep Vein Thrombosis: A Narrative Review, The American Journal of Medicine, Volume 128, Issue 7, 2015, Pages 722-738, ISSN 0002-9343, https://doi.org/10.1016/j.amjmed.2015.01.027. 
4. P. Holsteen, M. Meier, L. Brennan, et al., Safety and effectiveness of a risk-stratified venous thromboembolism prophylaxis algorithm in young people with cystic fibrosis, Thrombosis Research (2018), https://doi.org/10.1016/ j.thromres.2021.07.007

5. Hillegass E, Puthoff M, Frese EM, Thigpen M, Sobush DC, Auten B; Guideline Development Group. Role of Physical Therapists in the Management of Individuals at Risk for or Diagnosed With Venous Thromboembolism: Evidence-Based Clinical Practice Guideline. Phys Ther. 2016 Feb;96(2):143-66. doi: 10.2522/ptj.20150264. Epub 2015 Oct 29. PMID: 26515263. 